\title{
DISTRIBUTION OF THE PRESENT VALUE OF DIVIDEND PAYMENTS IN A LÉVY RISK MODEL
}

\author{
JEAN-FRANÇOIS RENAUD, * Université de Montréal \\ XIAOWEN ZHOU, ${ }^{* *}$ Concordia University
}

\begin{abstract}
In this short paper, we show how fluctuation identities for Lévy processes with no positive jumps yield the distribution of the present value of dividends paid until ruin in a Lévy insurance risk model with a dividend barrier.
\end{abstract}

Keywords: Lévy process; fluctuation theory; scale functions; insurance risk process; dividend barrier; dividend payments; time of ruin

2000 Mathematics Subject Classification: Primary 60G51

Secondary 91B30

\section{Introduction}

Intuitively, an insurance risk model with a dividend barrier describes the situation in which the premiums are paid out as dividends to shareholders whenever the surplus process reaches a certain level. A quantity of interest in this model with a constant barrier is the so-called present value of all dividends paid until the time of ruin. The distribution of this quantity was derived by Dickson and Waters [4] and by Gerber and Shiu [8] for the classical compound Poisson risk process, and then by Li [13] when the underlying process is the classical risk process perturbed by a Brownian motion. For more on the distribution of the dividend payments, see Dickson and Waters [4], Gerber and Shiu [8], Li [13], and the references therein.

Many risk processes are in fact special Lévy processes with no positive jumps. The classical compound Poisson risk process perturbed by a Brownian motion is one of them. More generally, some models have used the classical compound Poisson risk process perturbed by a Lévy process as their risk process. See, for instance, Furrer [6], Yang and Zhang [14], Huzak et al. [9], and Garrido and Morales [7]. Another risk process is considered by Klüppelberg et al. [11]: it is a Lévy process which drifts to infinity. For a nice interpretation of the Lévy-Itô decomposition of a Lévy process in the context of risk theory, see Klüppelberg and Kyprianou [10].

In this paper, we obtain explicit expressions for the moments of the present value of all dividends paid until ruin in an insurance Lévy risk model with a constant barrier. Our approach uses the solution of the two-sided exit problem for a spectrally negative Lévy process, i.e. a Lévy process with no positive jumps. For such risk processes, Kyprianou and Palmowski [12] have simultaneously derived the same results and even more general distributional quantities: see Theorem 1 and Corollary 1. Their methodology relies on Itô's excursion theory instead of fluctuation identities.

Received 13 September 2006; revision received 22 February 2007.

* Postal address: Département de Mathématiques et de Statistique, Université de Montréal, C.P. 6128, Succ. CentreVille, Montréal, Québec H3C 3J7, Canada. Email address: renaud@dms.umontreal.ca

** Postal address: Department of Mathematics and Statistics, Concordia University, 1455 de Maisonneuve Blvd W., Montréal, Québec H3G 1M8, Canada. Email address: xzhou@ mathstat.concordia.ca 


\section{A Lévy risk model and the exit problem}

Let $U=(U(t))_{t \geq 0}$ be a Lévy process with no positive jumps. The law of $U$ such that $U(0)=u$ will be denoted by $\mathrm{P}_{u}$ and the corresponding expectation by $\mathrm{E}_{u}$. Any readers not familiar with Lévy processes are referred to Bertoin [1] for more details.

\subsection{Exit from a finite interval and the scale functions}

The following material is mostly taken from Bertoin [2]. As the Lévy process $U$ has no positive jumps, its Laplace transform is given by

$$
\mathrm{E}_{0}\left[\mathrm{e}^{\lambda U(t)}\right]=\mathrm{e}^{t \psi(\lambda)},
$$

for $\lambda \geq 0$ and $t \geq 0$. In this case, the Laplace exponent $\psi$ is convex and

$$
\lim _{\lambda \rightarrow \infty} \psi(\lambda)=\infty
$$

Thus, we can define its right-inverse function $\Phi:[0, \infty) \rightarrow[0, \infty)$ by

$$
\psi(\Phi(\lambda))=\lambda, \quad \lambda \geq 0 .
$$

We now define the so-called scale functions $\left\{W_{q} ; q \geq 0\right\}$ of the process $U$. For each $q$, $W_{q}:[0, \infty) \rightarrow[0, \infty)$ is the unique, strictly increasing and continuous function with Laplace transform

$$
\int_{0}^{\infty} \mathrm{e}^{-\lambda x} W_{q}(x) \mathrm{d} x=\frac{1}{\psi(\lambda)-q},
$$

where $\lambda>\Phi(q)$. Sometimes the scale functions are denoted by $W^{(q)}$.

If we were interested in the two-sided exit problem, then the scale functions would arise naturally. Indeed, let $a$ be a positive real number and define

$$
T_{(0, a)}=\inf \{t \geq 0 \mid U(t) \notin(0, a)\} .
$$

When the process $U$ starts from within the interval, i.e. when $U(0)=u$ for $u \in(0, a)$, the random time $T_{(0, a)}$ is the first exit time of $U$ from this interval. Its Laplace transform on the event where the process $U$ leaves the interval at the upper boundary is given by

$$
\mathrm{E}_{u}\left[\mathrm{e}^{-q T_{(0, a)}} ; U\left(T_{(0, a)}\right)=a\right]=\frac{W_{q}(u)}{W_{q}(a)}, \quad q \geq 0 .
$$

Consequently, when $q=0$,

$$
\mathrm{P}_{u}\left\{U\left(T_{(0, a)}\right)=a\right\}=\frac{W_{0}(u)}{W_{0}(a)} .
$$

Throughout the paper we will assume that either the sample paths of $U$ have unbounded variation or the Lévy measure of $U$ is absolutely continuous with respect to the Lebesgue measure. The first condition is satisfied if $U$ has a Gaussian component. Under one of these assumptions, the scale functions $W_{q}$ are differentiable; see Doney [5] or Chan and Kyprianou [3]. The differentiability of the scale functions will be useful in what follows. The scale functions are also differentiable if we impose different assumptions on the spectrally negative Lévy process $U$; see Chan and Kyprianou [3] for more details. 


\subsection{A Lévy risk model with a dividend barrier}

Let $b$ be a positive real number. If the process $U$ starts from $u \in(0, b)$, then we define

$$
D(t)=\sup _{0 \leq s \leq t}(U(s)-b)^{+}
$$

and

$$
U_{b}(t)=U(t)-D(t)
$$

for every $t \geq 0$. One can think of $U_{b}=\left(U_{b}(t)\right)_{t \geq 0}$ as the surplus process of an insurance company that pays out, as dividends, any capital above the level $b$. Thus, $D(t)$ is the total amount of dividends paid up to time $t$.

We define the ruin time of this risk model with a barrier by

$$
T=\inf \left\{t \geq 0 \mid U_{b}(t) \leq 0\right\}
$$

Let $\delta$ be a nonnegative real number. Our main goal is to compute the distribution of

$$
D=\int_{0}^{T} \mathrm{e}^{-\delta t} \mathrm{~d} D(t)
$$

This quantity is the present value of all dividends paid until the time of ruin $T$, where $\delta$ can be interpreted as the force of interest. If $\delta=0$, then $D=D(T)$. The law of $D$ will be expressed in terms of the scale functions $\left\{W_{q} ; q \geq 0\right\}$.

Finally, for each $k \geq 1$, we introduce

$$
V_{k}(u)=\mathrm{E}_{u}\left[D^{k}\right]
$$

the $k$ th moment of $D$ when the process $U$ starts from $u$. The mean value of $D$ was previously computed by Zhou [15].

\section{The moments when starting from $b$}

First, we compute the moments of $D$ when $U$ starts from $b$, the barrier level.

Proposition 1. For $k \geq 1$,

$$
V_{k}(b)=k ! \prod_{i=1}^{k} \frac{W_{i \delta}(b)}{W_{i \delta}^{\prime}(b)} .
$$

Proof. Firstly, we obtain a lower bound for $V_{k}(b)$. For each $n \geq 1$, we introduce an exit time $T_{n}$ defined by

$$
T_{n}=\inf \left\{t \geq 0 \mid U(t) \notin\left(\frac{1}{n}, b+\frac{1}{n}\right)\right\} .
$$

As $U$ has no positive jumps, we have

$$
V_{k}(b)=\mathrm{E}_{b}\left[D^{k} ; U\left(T_{n}\right) \leq \frac{1}{n}\right]+\mathrm{E}_{b}\left[D^{k} ; U\left(T_{n}\right)=b+\frac{1}{n}\right] .
$$


As $T_{n}$ is strictly less than $T$ on the event $\left\{U\left(T_{n}\right)=b+1 / n\right\}$, using the Binomial Theorem and the strong Markov property at time $T_{n}$, we obtain

$$
\begin{aligned}
& \mathrm{E}_{b}\left[D^{k} ; U\left(T_{n}\right)=b+\frac{1}{n}\right] \\
& \quad=\sum_{j=0}^{k} C_{j}^{k} \mathrm{E}_{b}\left[\mathrm{e}^{-(k-j) \delta T_{n}}\left(\int_{0}^{T_{n}} \mathrm{e}^{-\delta t} \mathrm{~d} D(t)\right)^{j} ; U\left(T_{n}\right)=b+\frac{1}{n}\right] V_{k-j}(b),
\end{aligned}
$$

where $C_{j}^{k}=\left(\begin{array}{l}k \\ j\end{array}\right)$. Applying the integration by parts formula to $\int_{0}^{T_{n}} \mathrm{e}^{-\delta t} \mathrm{~d} D(t)$, using the fact that $D\left(T_{n}\right)=1 / n$ on $\left\{U\left(T_{n}\right)=b+1 / n\right\}$, and once again using the Binomial Theorem, we obtain

$$
\begin{aligned}
V_{k}(b)= & \mathrm{E}_{b}\left[D^{k} ; U\left(T_{n}\right) \leq \frac{1}{n}\right] \\
& +\sum_{j=0}^{k} C_{j}^{k} V_{k-j}(b) \sum_{i=0}^{j} C_{i}^{j} \delta^{j-i}\left(\frac{1}{n}\right)^{i} e(i, j, k ; n),
\end{aligned}
$$

where

$$
e(i, j, k ; n)=\mathrm{E}_{b}\left[\mathrm{e}^{-(k-j+i) \delta T_{n}}\left(\int_{0}^{T_{n}} \mathrm{e}^{-\delta t} D(t) \mathrm{d} t\right)^{j-i} ; U\left(T_{n}\right)=b+\frac{1}{n}\right] .
$$

Keeping only the terms for $j=i=0$ and $j=i=1$, and using the fluctuation identity of (1), we obtain

$$
V_{k}(b) \geq V_{k}(b) \frac{W_{k \delta}(b)}{W_{k \delta}(b+1 / n)}+k V_{k-1}(b) \frac{1}{n} \frac{W_{k \delta}(b)}{W_{k \delta}(b+1 / n)} .
$$

Secondly, we obtain an upper bound for $V_{k}(b)$. For each $n \geq 1$, we now define a new exit time $T_{n}^{\prime}$ by

$$
T_{n}^{\prime}=\inf \left\{t \geq 0 \mid U(t) \notin\left(0, b+\frac{1}{n}\right)\right\} .
$$

For each $T_{n}^{\prime}$, we also define

$$
S_{n}=\inf \left\{t \geq T_{n}^{\prime} \mid U_{b}(t) \leq 0\right\} .
$$

This is the time of ruin in the model with a barrier when $U$ starts at the random time $T_{n}^{\prime}$. Then, using similar arguments as before, for instance the strong Markov property at time $T_{n}^{\prime}$, we obtain

$$
\begin{aligned}
V_{k}(b) \leq & \mathrm{E}_{b}\left[\left(\int_{0}^{T_{n}^{\prime}} \mathrm{e}^{-\delta t} \mathrm{~d} D(t)\right)^{k} ; U\left(T_{n}^{\prime}\right) \leq 0\right] \\
& +\sum_{j=0}^{k} C_{j}^{k} V_{k-j}(b) \sum_{i=0}^{j} C_{i}^{j} \delta^{j-i}\left(\frac{1}{n}\right)^{i} e^{\prime}(i, j, k ; n),
\end{aligned}
$$

where

$$
e^{\prime}(i, j, k ; n)=\mathrm{E}_{b}\left[\mathrm{e}^{-(k-j+i) \delta T_{n}^{\prime}}\left(\int_{0}^{T_{n}^{\prime}} \mathrm{e}^{-\delta t} D(t) \mathrm{d} t\right)^{j-i} ; U\left(T_{n}^{\prime}\right)=b+\frac{1}{n}\right] .
$$


Before going any further, we provide estimates on the terms involved in the upper bound of (5):

- Let $l$ be any positive integer. Using (2), we obtain

$$
\begin{aligned}
\mathrm{E}_{b}\left[\left(\int_{0}^{T_{n}^{\prime}} \mathrm{e}^{-\delta t} \mathrm{~d} D(t)\right)^{l} ; U\left(T_{n}^{\prime}\right) \leq 0\right] & \leq\left(\frac{1}{n}\right)^{l} \mathrm{P}_{b}\left\{U\left(T_{n}^{\prime}\right) \leq 0\right\} \\
& =\left(\frac{1}{n}\right)^{l}\left(1-\frac{W_{0}(b)}{W_{0}(b+1 / n)}\right) .
\end{aligned}
$$

- Let $l$ and $m$ be any nonnegative integers. Then,

$$
\begin{aligned}
\mathrm{E}_{b}\left[\mathrm{e}^{-m \delta T_{n}^{\prime}}\left(\int_{0}^{T_{n}^{\prime}} \mathrm{e}^{-\delta t} D(t) \mathrm{d} t\right)^{l} ; U\left(T_{n}^{\prime}\right)=b+\frac{1}{n}\right] & \\
& \leq\left(\frac{1}{n}\right)^{l} \mathrm{E}_{b}\left[\left(\int_{0}^{T_{n}^{\prime}} \mathrm{e}^{-\delta t} \mathrm{~d} t\right)^{l} ; U\left(T_{n}^{\prime}\right)=b+\frac{1}{n}\right] \\
& \leq \begin{cases}\frac{W_{0}(b)}{W_{0}(b+1 / n)} & \text { if } l=0 ; \\
\left(\frac{1}{n}\right)^{l}\left(\frac{W_{0}(b)}{W_{0}(b+1 / n)}-\frac{W_{\delta}(b)}{\delta W_{\delta}(b+1 / n)}\right) & \text { if } l \geq 1 .\end{cases}
\end{aligned}
$$

As the scale functions are continuous, if $l \geq 1$, then we have

$$
\mathrm{E}_{b}\left[\left(\int_{0}^{T_{n}^{\prime}} \mathrm{e}^{-\delta t} \mathrm{~d} D(t)\right)^{l} ; U\left(T_{n}^{\prime}\right) \leq 0\right]=o\left(\frac{1}{n}\right),
$$

and

$$
\mathrm{E}_{b}\left[\mathrm{e}^{-m \delta T_{n}^{\prime}}\left(\int_{0}^{T_{n}^{\prime}} \mathrm{e}^{-\delta t} D(t) \mathrm{d} t\right)^{l} ; U\left(T_{n}^{\prime}\right)=b+\frac{1}{n}\right]=o\left(\frac{1}{n}\right),
$$

when $n$ goes to infinity. Consequently, if $j>i$,

$$
e^{\prime}(i, j, k ; n)=o\left(\frac{1}{n}\right) \text {. }
$$

This means that we have to deal with the terms for $j=i=0$ and $j=i=1$ in (5) carefully.

We now complete the proof. For $k \geq 1$, using the lower bound of (4), the upper bound of (5), the fluctuation identity of (1), and the previous estimates, we have

$$
V_{k}(b)=V_{k}(b) \frac{W_{k \delta}(b)}{W_{k \delta}(b+1 / n)}+k V_{k-1}(b) \frac{1}{n} \frac{W_{k \delta}(b)}{W_{k \delta}(b+1 / n)}+o\left(\frac{1}{n}\right) .
$$

Solving (6) for $V_{k}(b)$ and taking the limit, we obtain

$$
\begin{aligned}
V_{k}(b) & =\lim _{n \rightarrow \infty} \frac{W_{k \delta}(b+1 / n)}{n\left(W_{k \delta}(b+1 / n)-W_{k \delta}(b)\right)} k V_{k-1}(b) \frac{W_{k \delta}(b)}{W_{k \delta}(b+1 / n)} \\
& =\frac{W_{k \delta}(b)}{W_{k \delta}^{\prime}(b)} k V_{k-1}(b) .
\end{aligned}
$$

In the last line, we used the fact that, under our assumptions, the scale functions are differentiable. As $V_{0}(b)=1$, (3) follows. 


\section{The moments when starting from $u$}

Here is the main result of the paper, i.e. the moments of $D$ when $U$ starts from $u \in(0, b)$.

Proposition 2. For $k \geq 1$,

$$
V_{k}(u)=k ! \frac{W_{k \delta}(u)}{W_{k \delta}(b)} \prod_{i=1}^{k} \frac{W_{i \delta}(b)}{W_{i \delta}^{\prime}(b)} .
$$

Proof. Recall that $T_{(0, b)}=\inf \{t \geq 0 \mid U(t) \notin(0, b)\}$. As $T_{(0, b)}$ is strictly less than $T$ on the event $\left\{U\left(T_{(0, b)}\right)=b\right\}$, by the strong Markov property at time $T_{(0, b)}$, we obtain

$$
\begin{aligned}
V_{k}(u) & =\mathrm{E}_{u}\left[\left(\int_{T_{(0, b)}}^{T} \mathrm{e}^{-\delta t} \mathrm{~d} D(t)\right)^{k} ; U\left(T_{(0, b)}\right)=b\right] \\
& =\mathrm{E}_{u}\left[\mathrm{e}^{-k \delta T_{(0, b)}} ; U\left(T_{(0, b)}\right)=b\right] V_{k}(b) \\
& =\frac{W_{k \delta}(u)}{W_{k \delta}(b)} V_{k}(b) .
\end{aligned}
$$

The result then follows from Proposition 1.

\section{The Laplace transform}

As we have all the moments of $D=\int_{0}^{T} \mathrm{e}^{-\delta t} \mathrm{~d} D(t)$, we can make explicit the expression of its Laplace transform. We know, from Proposition 2 (or Proposition 1), that

$$
V_{1}(b)=\mathrm{E}_{b}\left[\int_{0}^{T} \mathrm{e}^{-\delta t} \mathrm{~d} D(t)\right]=\frac{W_{\delta}(b)}{W_{\delta}^{\prime}(b)} .
$$

Hence, when $\delta$ goes to infinity, $W_{\delta}(b) / W_{\delta}^{\prime}(b)$ decreases to zero.

Corollary 1. If $\delta>0$, then for every real number $\lambda$, the Laplace transform of $D$ exists and is given by

$$
\mathrm{E}_{u}\left[\mathrm{e}^{\lambda D}\right]=1+\sum_{k \geq 1} \lambda^{k} \frac{W_{k \delta}(u)}{W_{k \delta}(b)} \prod_{i=1}^{k} \frac{W_{i \delta}(b)}{W_{i \delta}^{\prime}(b)} .
$$

Proof. We first prove that

$$
\sum_{k \geq 0} \frac{|\lambda|^{k}}{k !} V_{k}(u)
$$

is finite. As $\delta>0$, from the remark preceding Corollary 1, we can choose large enough $j$ such that

$$
0 \leq|\lambda| \frac{W_{j \delta}(b)}{W_{j \delta}^{\prime}(b)}<1 .
$$

From (1) we also know that, for every $q \geq 0$,

$$
\frac{W_{q}(u)}{W_{q}(b)} \leq 1 .
$$


Then, for any $k \geq j$, we have

$$
\begin{aligned}
\frac{|\lambda|^{k}}{k !} V_{k}(u) & \leq \prod_{i=1}^{k}\left(|\lambda| \frac{W_{i \delta}(b)}{W_{i \delta}^{\prime}(b)}\right) \\
& \leq\left(|\lambda|^{j-1} \prod_{i=1}^{j-1} \frac{W_{i \delta}(b)}{W_{i \delta}^{\prime}(b)}\right)\left(|\lambda| \frac{W_{j \delta}(b)}{W_{j \delta}^{\prime}(b)}\right)^{k-j+1} .
\end{aligned}
$$

Therefore, the series in (7) is finite. The statement of the corollary follows from the monotone convergence theorem when $\lambda>0$, and from Lebesgue's dominated convergence theorem when $\lambda \leq 0$.

As already mentioned, if we assume that there is no force of interest, then the present value of all dividends paid until the time of ruin is equal to the total amount of dividends paid up to the time of ruin, i.e. if $\delta=0$, then $D=D(T)$.

Corollary 2. For $k \geq 1$,

$$
\mathrm{E}_{u}\left[D(T)^{k}\right]=k ! \frac{W_{0}(u)}{W_{0}(b)}\left(\frac{W_{0}(b)}{W_{0}^{\prime}(b)}\right)^{k},
$$

and then

$$
\mathrm{E}_{u}\left[\mathrm{e}^{-\lambda D(T)}\right]=1-\frac{\lambda W_{0}(u)}{W_{0}^{\prime}(b)+\lambda W_{0}(b)},
$$

for every $\lambda>-W_{0}^{\prime}(b) / W_{0}(b)$.

Observe that under $\mathrm{P}_{b}$, the moments and the Laplace transform of $D(T)$ are those of a random variable following an exponential distribution with mean $W_{0}(b) / W_{0}^{\prime}(b)$. Indeed, when $U$ starts from $b$, the Laplace transform of $D(T)$ is given by

$$
\mathrm{E}_{b}\left[\mathrm{e}^{-\lambda D(T)}\right]=\frac{W_{0}^{\prime}(b)}{W_{0}^{\prime}(b)+\lambda W_{0}(b)},
$$

for every $\lambda>-W_{0}^{\prime}(b) / W_{0}(b)$.

\section{Acknowledgements}

We wish to thank an anonymous referee for a careful reading of the paper that helped us improve Corollary 1 and for bringing to our attention the interesting paper by Kyprianou and Palmowski [12]. We would like to thank Hansjörg Albrecher who also brought that paper to our attention during the review process.

Partial funding in support of this work was provided by a doctoral scholarship of the Institut de Finance Mathématique de Montréal (IFM2), as well as by a Natural Sciences and Engineering Research Council of Canada (NSERC) grant.

\section{References}

[1] Bertoin, J. (1996). Lévy Processes. Cambridge University Press.

[2] Bertoin, J. (1997). Exponential decay and ergodicity of completely asymmetric Lévy processes in a finite interval. Ann. Appl. Prob. 7, 156-169. 
[3] Chan, T. and Kyprianou, A. E. (2006). Smoothness of scale functions for spectrally negative Lévy processes. Submitted.

[4] Dickson, D. C. M. And Waters, H. R. (2004). Some optimal dividends problems. ASTIN Bull. 34, 49-74.

[5] Doney, R. A. (2005). Some excursion calculations for spectrally one-sided Lévy processes. In Séminaire de Probabilités XXXVIII (Lecture Notes Math. 1857), Springer, Berlin, pp. 5-15.

[6] Furrer, H. (1998). Risk processes perturbed by $\alpha$-stable Lévy motion. Scand. Actuarial J. 1998, 59-74.

[7] Garrido, J. and Morales, M. (2006). On the expected discounted penalty function for Lévy risk processes. N. Amer. Actuarial J. 10, 196-218.

[8] Gerber, H. U. and Shiu, E. S. W. (2004). Optimal dividends: analysis with Brownian motion. N. Amer. Actuarial J. 8, 1-20.

[9] Huzak, M., Perman, M., Šikić, H. and VondračEK, Z. (2004). Ruin probabilities and decompositions for general perturbed risk processes. Ann. Appl. Prob. 14, 1378-1397.

[10] Klüppelberg, C. And Kyprianou, A. E. (2006). On extreme ruinous behaviour of Lévy insurance risk processes. J. Appl. Prob. 43, 594-598.

[11] Klüppelberg, C., Kyprianou, A. E. and Maller, R. A. (2004). Ruin probabilities and overshoots for general Lévy insurance risk processes. Ann. Appl. Prob. 14, 1766-1801.

[12] Kyprianou, A. E. And Palmowski, Z. (2007). Distributional study of de Finetti's dividend problem for a general Lévy insurance risk process. J. Appl. Prob. 44, 428-443

[13] LI, S. (2006). The distribution of the dividend payments in the compound Poisson risk model perturbed by a diffusion. Scand. Actuarial J. 2006, 73-85.

[14] Yang, H. And Zhang, L. (2001). Spectrally negative Lévy processes with applications in risk theory. Adv. Appl. Prob. 33, 281-291.

[15] Zhou, X. (2006). Discussion on 'On optimal dividend strategies in the compound Poisson model' (H. U. Gerber and E. S. W. Shiu). N. Amer. Actuarial J. 10, 79-84. 